Disponible en ligne : www.cahiersagricultures.fr

\title{
Diversité des dynamiques locales dans les oasis du Sud de la Tunisie
}

\author{
Irène Carpentier ${ }^{1,2, *}$ \\ ${ }^{1}$ Université Paris I Panthéon Sorbonne, laboratoire LADYSS, 5, rue Valette, 75012 Paris, France \\ ${ }^{2}$ Institut de recherche sur le Maghreb contemporain (IRMC), rue Mohamed Ali Tahar, Tunis, Tunisie
}

\begin{abstract}
Résumé - En Tunisie, les politiques de modernisation et de libéralisation mises en œuvre depuis l'Indépendance ont contribué à d'importants changements des conditions de pratique de l'agriculture et à une transformation des systèmes oasiens traditionnels. De nouvelles stratégies d'adaptations apparaissent élevage, tourisme local, spécialisation des cultures, démarche de qualité -, renouvelant les fonctions du territoire. Dans un contexte national de bouleversements sociopolitiques, des dynamiques contradictoires apparaissent : fragmentation du territoire et émergence d'initiatives collectives pour la transformation des modes de gestion, promotion du territoire local de l'oasis et intégration accrue à des logiques globales. L'analyse de ces dynamiques permet de souligner le déficit d'articulation entre les différents projets de développement oasiens, entre initiatives locales et projets de l'État et des bailleurs de fonds. Fondé sur une série d'enquêtes auprès des exploitants de Tozeur et de Gabès, cet article analyse la transformation des modes de gestion et de valorisation du territoire, comme autant de réponses aux crises de la mondialisation. Malgré une apparente disqualification dans les discours et les représentations, la durabilité des oasis ne relève pas tant de projets de conservation ou de développement, que de la force de dynamiques locales diversifiées, répondant aux fonctions plurielles du territoire. L'article souligne aussi les liens socialement différenciés entre pratiques territoriales et durabilité du système oasien, au-delà d'une simple sauvegarde du milieu.
\end{abstract}

Mots clés : durabilité / Tunisie / territoire / gestion des ressources / politique

Abstract - Diversity of local dynamics in the oases of southern Tunisia. In Tunisia, modernization and liberalization policies implemented since Independence contributed to significant changes in the conditions of farming practices. These policies induced changes in traditional oasis systems. New adaptation strategies appear, such as local tourism, crop specialization, quality approaches. These strategies renew the functions of the territory. In a national context of political upheaval, contradictory dynamics appear: land fragmentation and emergence of producer groups aiming to renew current management models, promotion of local territory and increasing integration to global logics. The analysis of these dynamics highlights the lack of articulation between the current development projects, especially between local initiatives and State- and donor-led projects. Based on surveys among farmers in Tozeur and Gabès, this article analyzes the transformation of forms of territorial management and valorization, as answers of the local oasis territory to the global crisis. In spite of an apparent disqualification in discourses and representations, the sustainability of oases does not relate so much to conservation or development projects as to the strength of diversified local dynamics, responding to the pluralistic functions of the territory. This paper also highlights the socially differentiated links between practices of territory and sustainability of the oasis, beyond a mere safeguarding of the environment.

Keywords: sustainability / Tunisia / territory / resource management / politics

\section{Introduction}

Les bouleversements politiques au Sud de la Méditerranée, et en Tunisie en particulier, qui ont inauguré le " printemps arabe ", ont mis en lumière les profondes fractures sociales

\footnotetext{
* Auteur de correspondance : i.carpentier88@gmail.com
}

et territoriales de ces pays. L'accent sur la revendication émergente d'un " nouveau modèle de développement ", aspirant à plus de justice sociale dans la gestion des ressources locales, trouve son origine dans les régions agricoles et rurales et " renvoie de manière générale à l'aggravation des inégalités sociospatiales qui ont accompagné les processus de développement, surtout depuis la fin des années $80 »$ (Gana, 2013). 
Cet engagement pour l'émergence d'un nouveau modèle, plus à même de répondre aux besoins des territoires locaux, s'inscrit dans ce que Pecqueur (2006) appelle le «tournant territorial de l'économie globale ». Les processus de « reterritorialisation de l'organisation locale du développement » se multiplient, comme " solution » aux crises de la mondialisation. La question de la durabilité des territoires, non seulement écologique, mais aussi économique et sociale, s'inscrit dans un processus situé localement, et historiquement contextualisé (Campagne et Pecqueur, 2014). Par " territoire ", nous n'entendons pas un " simple support contenant des ressources inventoriées », mais plutôt « une production sociale que ses habitants peuvent transformer, et rendre plus ou moins attractif et compétitif » (Théry, 2008). Les liens entre dynamiques locales et processus économiques globaux ont souvent été pensés en termes d'impacts sur le territoire, présenté comme "victime passive ». Nous proposons ici de repenser cette articulation comme génératrice de processus contradictoires, qui renforcent et simultanément « nient», les territoires locaux (ibidem).

Les oasis du Sud de la Tunisie constituent un exemple caractéristique de l'apparition des territoires locaux dans les débats publics sur le développement. Dans un contexte marqué d'une part par la crise des systèmes oasiens dans leurs formes traditionnelles, et d'autre part par la montée des revendications des acteurs oasiens pour la redéfinition des modes de gestion des ressources, ceux-ci s'organisent pour la mise en place de nouvelles stratégies de valorisation du territoire, plus adaptées aux spécificités locales. La multiplication de nouveaux projets, à l'échelle individuelle et collective, invite à ne pas négliger le rôle que jouent les stratégies d'adaptation des acteurs locaux dans les dynamiques de transformation des territoires et de formulation de nouvelles perspectives de développement dans l'espace local (D'Aquino, 2002).

Cet article analyse la transformation des modes de gestion et de valorisation du territoire, comme autant de réponses aux crises de la mondialisation, qui se sont traduites par l'aggravation des inégalités sociospatiales et par des conséquences sur le milieu dues à la surexploitation des ressources en eau et en terres. Il analyse les processus de reconfiguration du territoire, dans un contexte politique national de remise en question des modes de gestion des ressources. Selon l'hypothèse discutée ici, la durabilité des oasis ne relève pas tant de projets de conservation ou de développement que de la diversité des dynamiques locales, qui mobilisent des fonctions plurielles du territoire.

\section{Méthodes}

Les différentes analyses s'appuient sur une série d'enquêtes, réalisées entre 2011 et 2014 , où se combinent 60 questionnaires auprès de chefs d'exploitation oasiens pour la caractérisation des projets et initiatives, et 20 entretiens semi-directifs complémentaires auprès d'exploitants, travailleurs de l'oasis, employés et responsables de l'administration, et acteurs associatifs pour cerner la diversité des représentations et des discours sur la gestion et la valorisation des ressources. Elles sont également basées sur une collecte systématique des données à l'échelle du parcellaire, auprès des listes des irrigants des huit groupements de développement agricoles (GDA) oasiens de Gabès et Tozeur, et complétées

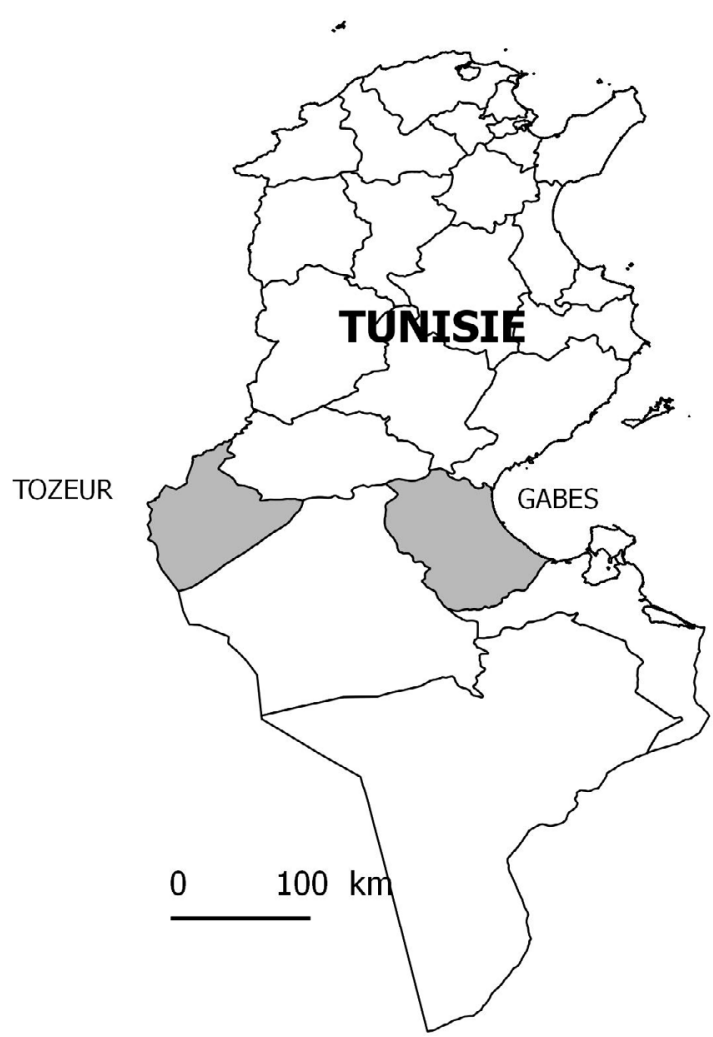

Fig. 1. Tozeur et Gabès, dans le Sud de la Tunisie.

Fig. 1. Tozeur and Gabès, in southern Tunisia.

avec l'aide des aiguadiers, par des informations sur les modes d'organisation du travail, les types de cultures, l'élevage, l'équipement, la pluriactivité... À ces données s'ajoute une revue systématique de l'action gouvernementale et des politiques et projets en matière de développement oasien.

Ce texte présente les exemples de Tozeur et de Gabès, deux oasis anciennes du Sud de la Tunisie, l'une continentale, l'autre littorale, symboliques des enjeux du développement du Sud de la Tunisie. Ces deux oasis permettent d'analyser la diversité de réponse des territoires oasiens aux projets et politiques de modernisation dont elles ont fait l'objet (Fig. 1-3).

\section{Crise des systèmes: les oasis, des territoires en mutation}

\subsection{Les oasis anciennes en crise}

Aujourd'hui, les rapports des pouvoirs publics et des bailleurs de fonds internationaux évoquent les oasis comme des espaces en crise. Le projet pour le « Développement durable des oasis », présenté par le ministère tunisien de l'Environnement et du développement durable en 2015, ou le rapport de la Deutsche Gesellschaft für Internationale Zusammenarbeit (GIZ) décrivent les oasis comme évoluant dans une "spirale vicieuse de dégradation » (ministère de l'Environnement, 2015), signe d'un système oasien « limité » (GIZ, 2013) et incapable de s'adapter à la conjoncture. 


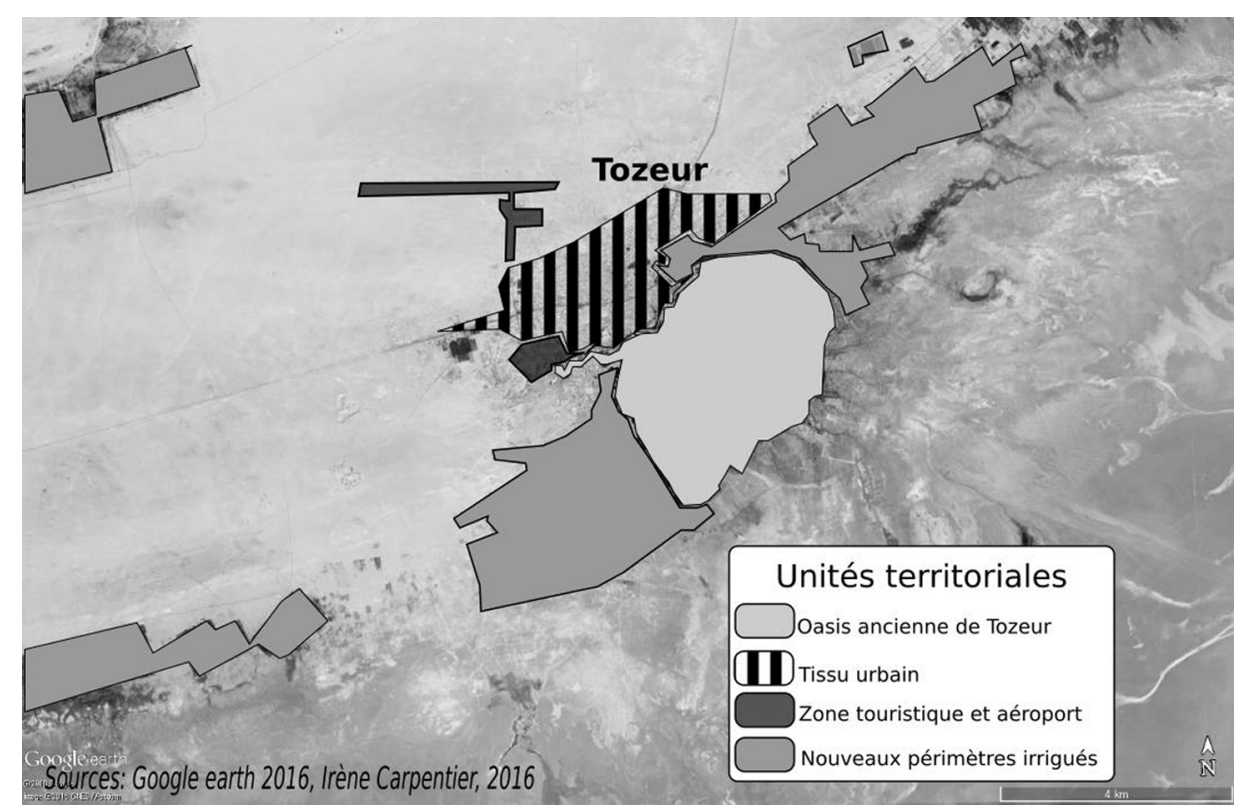

Fig. 2. Oasis de Tozeur.

Fig. 2. Tozeur oasis.

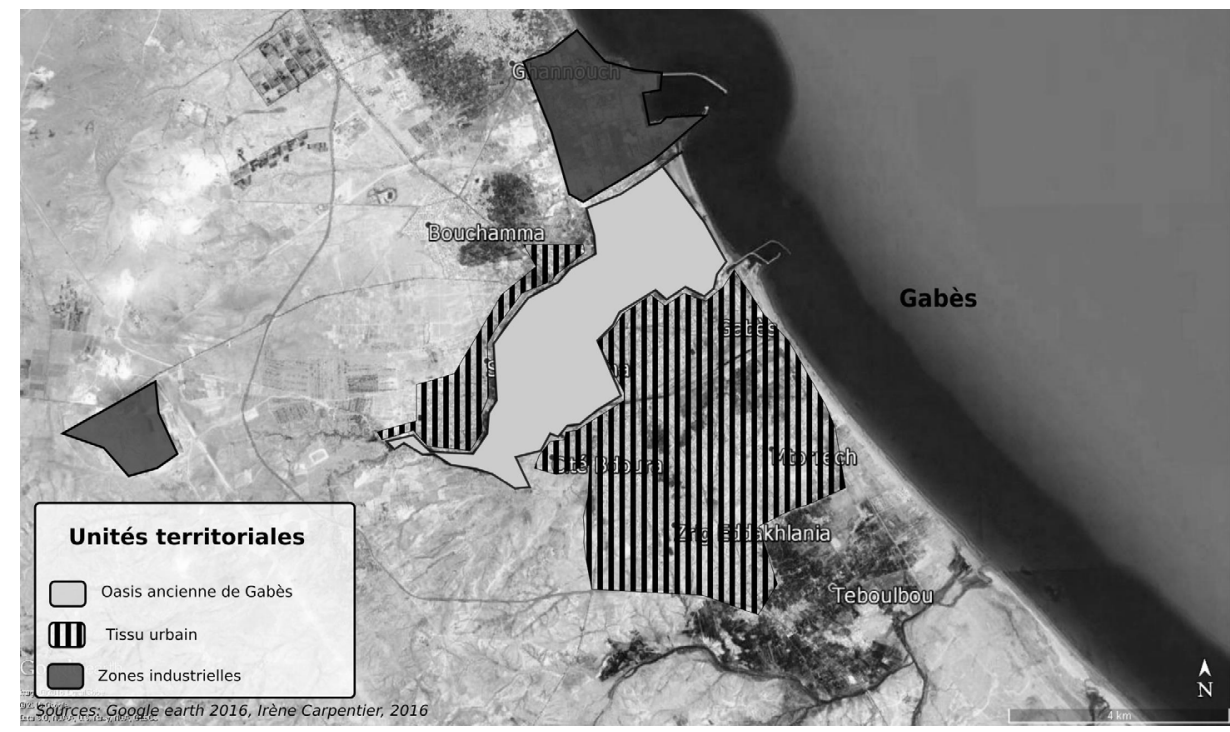

Fig. 3. Oasis de Gabès.

Fig. 3. Gabès oasis.

Cependant, « l'étonnant renouveau de l'agriculture saharienne » qu'évoque Côte (2002), ainsi que la capacité de résistance et d'adaptation de ces systèmes complexes, engagent le chercheur à préciser ce qu'est l'oasis aujourd'hui (Abdedayem et Veyrac-Ben Ahmed, 2014). Nous analysons ici les dynamiques des oasis anciennes, dont le paysage se caractérise par une forte densité de plantation et une importante biodiversité végétale. Même si ces caractéristiques ne sont plus toujours présentes, il n'est pas possible de les assimiler tout à fait aux nouveaux périmètres irrigués (ibidem). Nous nous focaliserons sur ces espaces dans la mesure où cela permet d'envisager les héritages sociohistoriques sur le temps long, et de mieux cerner les mutations dans la vocation des territoires oasiens.

\subsection{Des systèmes oasiens en transformation}

Les territoires oasiens font l'objet d'une double marginalisation : politique, avec un modèle national qui ne pense pas le développement dans ces territoires, et économique avec la mise en concurrence des espaces productifs pour l'accès aux ressources et au marché. Considérés par les pouvoirs publics comme structurellement inadaptés aux exigences de la modernité et du développement national (Battesti, 2005 ; Bras, 1996), les territoires oasiens sont gérés comme des périmètres irrigués comme les autres, «zones d'ombres » d'un développement national (Battesti, 2005; Gachet, 1987)) fondé sur la métropolisation et la littoralisation des activités 
(Belhedi, 1999). La diversité des fonctions du territoire oasien n'est que peu prise en compte (Abdedayem, 2015).

Sur le plan économique, les politiques de libéralisation qui se sont accélérées à la fin des années 1990 (Gana, 2008) contribuent à remettre en question la place des oasis anciennes dans le développement du sud de la Tunisie.

À Tozeur, dès l'Indépendance, et en continuité des politiques coloniales, l'extension des superficies irriguées en monoculture de palmiers Deglet Nour répond aux objectifs de politiques favorisant les cultures irriguées d'exportation, intensives. Dans les années 1990, le développement de l'activité touristique, avec un aéroport international, une zone d'hôtels, vise à diversifier le produit touristique national et désengorger le littoral.

Sur le littoral de Gabès, à partir des années 1970, c'est l'industrialisation qui doit garantir le développement régional. Il s'agit de valoriser les ressources minières à l'échelle nationale, avec la création d'une zone industrielle à côté du port commercial et d'une cimenterie dans l'intérieur du gouvernorat. Plus récemment, des périmètres irrigués se développent dans la plaine au sud et à l'ouest de Gabès.

Ces politiques donnent la priorité à l'ancrage de l'économie tunisienne dans la mondialisation et au développement des activités de services, au détriment des territoires oasiens historiques, qui apparaissent marginalisés et concurrencés dans l'accès aux ressources en eau, en terres, et au marché par les activités industrielles et de services, la fonction de loisir, et la rentabilité économique par les activités de tourisme.

\subsection{Un milieu sous pression}

L'assèchement des nappes, le morcellement foncier, l'urbanisation sont les dangers les plus fréquemment identifiés lorsqu'il s'agit de présenter les menaces qui pèsent sur l'oasis. Depuis les années 1990, les sources naturelles sont asséchées et les oasis, à Tozeur comme à Gabès, sont intégralement irriguées par des pompages dans la nappe souterraine (Battesti, 2005). L'agence foncière agricole de Gabès estime que l'oasis perd 10 ha par an depuis la fin des années 1980, au profit de l'urbanisation ; celle-ci apparaît comme la menace majeure pour la durabilité du milieu oasien de Gabès, en particulier dans sa partie littorale. À Tozeur, les responsables des GDA estiment qu'un tiers de la superficie totale de l'oasis est aujourd'hui abandonnée, menacée par un processus de désertification qui n'est pas enrayé. Depuis quelques années, des incendies ravagent des zones entières de l'oasis, en raison de la sécheresse et du mauvais entretien. Ainsi, 80 ha ont brûlé en 2013, selon les propos des responsables d'un GDA à Tozeur en 2014.

\section{4 Émergences de nouvelles opportunités: différenciation des trajectoires oasiennes et durabilité}

Le constat de crise des systèmes oasiens pose la question non seulement de la durabilité du milieu oasien, mais plus largement de sa capacité à se maintenir en territoire. Malgré les difficultés issues des profondes transformations de leur environnement économique et politique, les territoires oasiens de Tozeur et de Gabès sont des exemples de dynamiques internes de résistance. Présenter cette différenciation des choix stratégiques de mise en valeur au sein d'une typologie synthétique permet de souligner le potentiel d'adaptabilité des exploitants (Carpentier et Gana, 2017).

\subsection{Diversification des systèmes de culture}

Sur des propriétés aux superficies réduites et fortement morcelées, les petits exploitants, qui représentent $70 \%$ des exploitants à Tozeur et $63 \%$ à Gabès, pour respectivement 35 et $49 \%$ des terres (Carpentier et Gana, 2017), développent des systèmes aux cultures arboricoles, maraîchères, fourragères, diversifiées et étagées, associées au petit élevage. L'objectif pour les exploitants reste de garantir la reproduction du système et des exploitations, en optimisant un patrimoine familial. Les pratiques héritées des savoir-faire traditionnels greffe d'arbustes, association de cultures, jachère - ou les méthodes d'économie d'eau, permettent à ces exploitants de s'adapter aux contraintes d'accès à l'eau, de salinité des sols, et de commercialisation. L'autoconsommation reste une fonction non négligeable. À Tozeur, ce sont les khammes, métayers payés au cinquième de la récolte des dattes, qui ont à disposition les étages arboricoles et maraîchers, et contribuent au maintien de la diversité culturale et de la fonction nourricière. Le paysage de ces exploitations est richement diversifié, avec l'introduction de cultures peu courantes dans les oasis, comme l'aubergine ou le poireau, afin de minimiser les risques de concurrence sur le marché.

Ces petits exploitants, parmi les plus modestes de l'oasis, sont fortement soumis aux aléas de la conjoncture économique et politique. Ainsi, dans un contexte d'instabilité politique, où les rapports de force hérités sont mis en question, la durabilité sociale de cette catégorie n'est pas garantie. D'autre part, la rentabilité économique est rarement au rendez-vous (Romdhane et Abdeladhim, 2008) et les problèmes d'accès aux ressources en eau et en terres rendent fragile ce type d'exploitants, pourtant majoritaire.

\subsection{Diversification des systèmes de production}

Tournée massivement vers l'intensification et la spécialisation, avec l'élevage bovin en particulier, une autre catégorie d'exploitations, qui représente près d'un quart des exploitants à Gabès et $5 \%$ à Tozeur, a profité des programmes étatiques pour l'encouragement de l'élevage bovin intra-oasien. Les revenus quotidiens permettent une relative insertion des producteurs dans les marchés urbains régionaux par la mise en circulation quotidienne des productions. L'ensemble de l'exploitation s'oriente vers la satisfaction des besoins du cheptel, contribuant à la dégradation de l'étagement du paysage, avec la quasi-disparition de l'étage arboricole au profit d'une spécialisation dans les cultures fourragères. De taille relativement supérieure à la moyenne $(0,3$ ha à $0,6 \mathrm{ha})$, ces exploitations sont fondées sur un objectif de rentabilité économique, pas toujours atteint, du fait de l'inflation des prix des aliments pour les animaux depuis 2011, et de la fragilité des bêtes, souvent de races importées.

Par ailleurs, de nombreuses études interrogent la pertinence de l'élevage bovin oasien (Abaab, 2004 ; Romdhane et Abdeladhim, 2008) : élevage presque « hors sol», reposant sur 
un système d'alimentation dépendant des importations, fortement consommateur en eau et en terres, et qui induit l'artificialisation des terres avec la construction d'étables.

\subsection{Le marketing territorial : l'activité de loisir dans l'oasis}

Une autre forme de projet consiste en l'introduction d'activités de services, projets de tourisme et loisirs, au sein de l'oasis ancienne. À Tozeur, 18 projets, souvent des parcs-cafés, sont implantés dans l'oasis. Il y en a 12 à Gabès. S'ils ne sont qu'une minorité d'exploitants à faire ce choix $-2 \%$ à Tozeur et $0,4 \%$ à Gabès, pour respectivement 4,4 et $2,2 \%$ des terres (enquêtes 2013), cela n'en reste pas moins significatif dans la mesure où cela contribue à modifier profondément les activités et fonctions de l'oasis. Les plus grands propriétaires de l'oasis développent leurs projets dans les zones les plus accessibles, le long de la " route touristique ». Souvent, ils ont travaillé à l'étranger et sont actifs dans d'autres secteurs. Ils bénéficient d'une infrastructure hydraulique complémentaire au réseau collectif, sous la forme de bassin ou de puits individuel, en particulier à Tozeur où les plus grands exploitants sont équipés (Carpentier et Gana, 2014).

Dans le contexte de crise touristique que traversent les oasis, du fait de la situation sécuritaire nationale, ces espaces de loisirs sont de plus en plus investis par la population locale. Dans ces exploitations, la fonction productive de l'activité agricole n'est plus une priorité, et ce au profit de la fonction esthétique. Le territoire oasien devient espace de loisir, au paysage recréé selon les canons d'un âge d'or idéalisé (Battesti, 2005), et se constitue en espace de sociabilité pour les urbains. L'orientation vers des activités de services est un moyen pour saisir les opportunités d'un marché toujours plus restreint, tant sur le plan agricole que touristique. Cette dynamique, destinée à une clientèle internationale mais aussi locale, peut être interprétée comme renvoyant à un processus de tertiarisation du territoire. Marginalisées pour leur faiblesse productive, les oasis, par cet intermédiaire, se réinsèrent dans l'espace régional.

\subsection{L'agrobusiness oasien}

Une tendance marquante de l'évolution des systèmes oasiens est le développement d'exploitations spécialisées dans la production de dattes Deglet Nour dans les oasis anciennes. À Tozeur, des projets se développent dans une logique entrepreneuriale au moyen de forts investissements. Au sein d'une infrastructure moderne complète (électricité, puits, bassins, séguias en PVC ou bétonnées, bâti), les projets visent la conquête de marchés d'exportation. L'accès à l'eau, autonome, est assuré par la présence d'un puits sur la parcelle. La spécialisation des cultures débouche sur la monoculture en Deglet Nour. Les exploitations sont parmi les plus grandes (supérieures à $1,5 \mathrm{ha}$ ), et sont situées le long des routes. À Tozeur, c'est dans la zone centrale de Wassat que l'on trouve le plus ce genre d'exploitation, comme celle de L., dans la zone nommée Touareg. Propriétaire de 4 ha dont il a hérité, sur lesquels sont plantés 500 palmiers dont 400 de variété Deglet Nour, il confie la récolte sur pied à une société privée qui se charge de l'exportation. L'exploitation est dotée d'un puits qui permet de garantir une certaine autonomie dans l'accès à l'eau.
Situé dans les oasis anciennes, ce type d'exploitation reproduit un modèle déjà communément présent dans les nouveaux périmètres, mais qui est nouveau dans les périmètres anciens. Dans l'oasis ancienne, ces exploitations concurrencent fortement les autres pour l'accès aux ressources en eau et en sol. La rentabilité agricole y est importante (Battesti, 2005). Ces projets sont pleinement ancrés dans des logiques d'intégration à des marchés considérés comme porteurs par les pouvoirs publics et les sociétés d'exportation. Le fort dynamisme des opérateurs économiques dattiers de la zone (Horchani, Nouri...), qui produisent, transforment, stockent et commercialisent les dattes, permet d'entretenir le développement de la filière (Bachta et al., 2006). Ces sociétés d'exportation s'appuient sur des réseaux internationaux de clientèle, en particulier en Europe, qui se diversifient vers l'Amérique du Nord ou les Émirats arabes unis. Cet ancrage dans le marché mondial participe d'une consolidation des positions dominantes de ces exploitants dans la compétition pour l'accès aux ressources.

Souvent, ces agro-entrepreneurs revendiquent aussi leur contribution à la durabilité du milieu oasien dans la mesure où les projets permettent d'éviter l'abandon des terres et préservent la fonction agricole. De plus, ceux-ci prétendent également contribuer à la promotion de filières alternatives. Ainsi, de nombreuses exploitations ont fait le choix de la conversion en agriculture biologique. Cela permet de saisir de nouvelles opportunités en optant pour une "démarche de qualité ", répondant en priorité à une demande internationale. Ces stratégies sont promues par les institutions. À Tozeur, le ministère de l'Agriculture prévoit officiellement une conversion en bio de l'ensemble des palmeraies à l'horizon 2030, afin d'optimiser la rente que constitue la Deglet Nour, en priorité dans les nouveaux périmètres irrigués, mais aussi auprès de ces exploitations en monoculture des espaces oasiens anciens, pour à terme labelliser l'ensemble des oasis.

\subsection{Acteurs associatifs et promotion de démarches de qualité}

La démarche de qualité se décline aussi sous la forme de projets de labellisation, de développement des filières biologiques, d'activités éco-touristiques, en lien avec l'héritage d'une agriculture familiale multifonctionnelle. Cette dynamique participe d'un projet de réhabilitation du milieu oasien, par la mobilisation des savoir-faire traditionnels et la promotion de pratiques agro-écologiques, comme le compostage ou la production de semences anciennes. Portés non pas par des entrepreneurs individuels, mais par des organisations collectives composées de militants pluriactifs, souvent de classes socioprofessionnelles supérieures, à Gabès et Tozeur, ces projets s'adressent aux petits exploitants et les y associent.

Les actions visent la mise en valeur et la préservation du paysage oasien, caractérisé par des cultures diversifiées et étagées. La conquête d'autonomie dans la gestion des ressources est un des enjeux principaux. Le jardin oasien est promu comme outil pédagogique pour expérimenter de nouvelles pratiques de valorisation de l'oasis, comme à Chenini Gabès avec la mise en place d'un " jardin de la biodiversité ». La mise en place de filières « bio » (les grenades 
à Chenini Gabès) ou la transformation des produits sont des projets visant à consolider un réseau de petits agriculteurs autour d'un modèle alternatif de mise en valeur des territoires oasiens. Le développement d'activités de services liées au tourisme, à la culture ou à l'artisanat dans l'oasis est au cœur de ces stratégies de diversification. La durabilité environnementale est promue par les acteurs associatifs comme base de la durabilité économique et sociale.

\subsection{Cohabitation des stratégies d'adaptation et durabilité oasienne : fragmentation et multifonctionnalité des territoires}

Cette diversité des initiatives souligne à quel point les impacts de la mondialisation sur les territoires locaux sont loin d'être uniformes. Ainsi, la différenciation des dynamiques de valorisation et d'adaptation aux crises du développement dans les oasis est à l'origine d'une fragmentation du territoire, sur le plan social, économique et paysager, qui pose la question de la transformation des modèles de mise en valeur du territoire. L'émergence de tendances contradictoires dans les projets oasiens - spécialisation, intensification ou diversification témoigne de cette fragmentation des dynamiques de développement du territoire.

La durabilité des systèmes oasiens passe alors par la cohabitation d'initiatives diversifiées, plutôt que par la multiplication de programmes de sauvegarde à l'échelle de l'ensemble des oasis. En effet, chaque forme de valorisation présentée dans cette typologie apporte une réponse socialement différenciée aux contraintes de l'oasis, entre promotion d'une petite agriculture familiale diversifiée, consolidation d'exploitations dominantes, et maintien d'exploitations de taille moyenne. Cette diversification ne va pas sans confrontations, et un certain nombre d'enjeux apparaissent conflictuels, comme l'organisation de la main-d'œuvre agricole, l'accès aux ressources en eau ou en terres. La question n'est alors pas tant celle du maintien des systèmes oasiens que celle des critères de ce maintien : entre intégration régionale, autonomie de la production, gestion des ressources, organisation sociale... Différentes fonctions de l'oasis sont sollicitées, répondant aux injonctions internationales de multifonctionnalité des territoires comme conditions de durabilité des espaces ruraux.

\section{Les oasis tunisiennes : un territoire d'action au cœur d'une redéfinition des modèles de développement}

À la lumière de ces analyses, il est nécessaire de préciser notre définition du territoire d'action. Ainsi, le territoire peut être défini avant tout comme un « système d'action [...] instrument efficace de la réactivation des liens sociaux ». En effet, « il pose de manière très réaliste [...] la question, dans un espace social donné, du partage des lieux, des ressources et des pouvoirs »(Di Méo, 2006). De plus, ces « nouveaux territoires du social peuvent constituer un levier efficace du développement durable, parce que ce sont des espaces commodes de convention, de contrat et donc de gouvernance » (ibidem). Cette approche du territoire comme système d'action nous semble particulièrement intéressante pour les oasis tunisiennes.

\subsection{Discours émergents et programme de promotion des oasis : un besoin consensuel de durabilité}

Alors que les dynamiques internes aux oasis permettent d'observer des stratégies de mise en valeur socialement différenciées, les discours pour leur sauvegarde continuent à les présenter comme homogènes et statiques. Cependant, là aussi, les discours se recomposent.

Depuis 2011 et l'émergence d'un débat sur les modèles de développement, les territoires oasiens anciens apparaissent dans les discours des différents acteurs impliqués - exploitants, groupements de producteurs, associations, administration, bailleurs de fonds - comme un « bien commun » que tous s'accordent à protéger (GIZ et ministère de l'Environnement, 2012). Ainsi, la « stratégie nationale de sauvegarde », ou le « plan pour la gestion durable » des oasis par les pouvoirs publics et les bailleurs de fonds symbolisent la production de ce discours présentant les oasis comme objets privilégiés des politiques de développement durable.

Les oasis font l'objet de labellisations visant à protéger leurs caractères exceptionnels et universels. L'oasis de Gabès a fait l'objet d'une démarche pour être classée, et est aujourd'hui placée sur la liste indicative pour un classement au patrimoine mondial de l'Unesco. Le programme SIPAM (Système ingénieux du patrimoine agricole mondial) de la FAO, vise à « labelliser » les oasis anciennes comme systèmes porteurs de développement durable et de « coadaptation entre une société et son environnement» (FAO, 2008).

À l'échelle nationale, les programmes pour la protection des oasis se sont également multipliés, avec le soutien des bailleurs de fonds internationaux. En 2010-2011, le PNUD apporte un appui au Programme de microfinancement du Fonds pour l'environnement mondial (PMF/FEM) dans les oasis de Tunisie. En 2013, la GIZ, en partenariat avec le ministère de l'Environnement, soutient le projet : les oasis de Tunisie à protéger contre la dégradation et les effets du changement climatique (GIZ et ministère de l'Environnement tunisien, 2012). En 2014, c'est la Banque mondiale qui finance le Oases ecosystems and livelihoods project. En 2015, le FEM appuie le ministère de l'Environnement dans son Plan d'action de la stratégie de développement durable des oasis en Tunisie (FEM et ministère de l'Environnement, 2015; Ministère de l'Environnement et du Développement durable tunisien et Direction générale de l'environnement et de la qualité de la vie, 2015).

L'ensemble de ces initiatives inscrit les territoires oasiens dans un cadre global, celui des sites du patrimoine mondial à sauvegarder, et redéfinit la contribution des oasis au développement régional et national. Ainsi, le ministère de l'Environnement évoque la nécessaire mise en place d'un « développement durable participatif » dans les oasis. Ces projets et discours, marquant une attention accrue à la sauvegarde des oasis, répondent avant tout à des injonctions extérieures de «durabilité » et de « bonne gouvernance », ne prenant finalement que peu en compte les initiatives locales, pourtant au cœur des transformations des espaces oasiens, et la diversité d'acteurs aux intérêts divergents, qui ne maîtrisent et ne s'insèrent pas tous de la même façon dans ces discours de gestion durable et participative. 


\subsection{Enjeux locaux et boom associatif, l'oasis comme territoire d'action}

Si le plaidoyer à l'échelle nationale et internationale en faveur des oasis apparait aujourd'hui consensuel, il ne faut pas négliger qu'il s'appuie sur l'affirmation d'une scène locale associative diversifiée après la Révolution (Carpentier et Gana, 2016). Ce "système d'actions » permet la réactivation dans l'espace oasien de liens sociaux, entre dynamiques locales et pouvoirs publics ou bailleurs de fonds, et s'organisent autour des questions de partage des ressources, des pouvoirs et des lieux (Di Méo, 2006).

À Gabès, l'essentiel de la dynamique associative se structure autour de la remise en cause des politiques industrielles, d'un militantisme environnemental et d'une volonté d'animation culturelle et sociale. En plus d'un renouvellement du bureau de l'ancienne association de sauvegarde de l'oasis, plusieurs nouvelles associations sont apparues après la Révolution, avec pour objectif la protection et valorisation de l'oasis. Ainsi, dès 2011, des associations de protection de l'environnement et d'animation locale sont créées : Gabès Action, SOS Environnement Gabès et l'Association formes et couleurs oasiennes (AFCO). En 2012 est créée l'Association tunisienne pour l'environnement et la nature de Gabès, et en 2013, l'Association de protection de l'oasis de Chott Essalem. Par ailleurs, des mouvements de lutte contre la pollution - stop pollution en 2012, green future en 2015 - ont émergé sur la scène locale, défendant, qui l'opposition frontale et le rejet de l'activité industrielle, qui une négociation avec le groupe chimique pour la réforme $\mathrm{du}$ modèle industriel. Ils organisent régulièrement des " évènements » pour redéfinir les politiques industrielles et de développement.

À Tozeur, une dynamique similaire est à l'œuvre. L'Association de sauvegarde de la Medina de Tozeur (ASMT), la plus ancienne et la plus dynamique sur les questions touristiques et de développement local, a renouvelé son bureau ; ses membres - universitaires, professeurs et jeunes diplômés - tentent de redéfinir les modèles touristiques à Tozeur, en créant des circuits pédagogiques dans la vieille ville et l'oasis. Éco-logement, restauration bio, les initiatives associatives redéfinissent le tourisme oasien au profit de la sauvegarde du capital culturel et paysager de l'oasis. De ce fait, une proposition alternative au modèle classique se constitue, pénétrant faiblement dans l'oasis et ses spécificités. Après la Révolution, de nouvelles associations de sauvegarde de l'oasis, fondées en 2011, visent à renouveler les pratiques de l'oasis et à s'attaquer aux problèmes fonciers. L'objectif est de promouvoir la richesse biologique de l'oasis de Tozeur, comme base d'une nouvelle valorisation du patrimoine écologique de l'oasis. L'association "Ruche de la citoyenneté » travaille sur la corruption des associations d'irrigation et la redéfinition des modes de gestion des ressources.

L'émergence de collectifs locaux pour la promotion et la protection des oasis reflète cette volonté de se saisir des problématiques du développement oasien et d'en redéfinir les conditions. Au travers de ces initiatives et projets, l'oasis apparaît comme un lieu privilégié de mobilisation collective. Les enjeux du développement oasien s'inscrivent dans un système d'action en recomposition, où un espace de dialogue s'est ouvert entre des acteurs aux intérêts divergents.

\subsection{Entre alternatives locales et débats politiques, des modèles en question}

La promotion des oasis comme espace patrimonial d'action par les acteurs locaux et les pouvoirs publics correspond à la tentative d'incarner une alternative locale pour répondre aux enjeux du développement régional.

Les dynamiques de différenciation sociale et de diversification des fonctions, si elles font des oasis un espace privilégié de l'analyse des recompositions des modèles de gestion, n'empêchent pas la reproduction de pratiques héritées. En effet, des divergences politiques existent sur les modalités de partage et de redistribution des ressources. La justice sociale, au cœur des revendications issues de la Révolution, n'apparaît que rarement dans les «plans de sauvegarde et de développement durable ». Cette déconnection, alliée à une certaine difficulté des acteurs locaux à formuler des alternatives organisées, contribue à complexifier l'articulation entre dynamiques locales et politiques de développement cohérentes. Ainsi, comme le rapporte un membre de Green Future: «La protection des oasis, c'est une noble tâche. Ce qui manque, ce sont les propositions de solutions. Le problème est purement politique. [...] Avant les élections de 2011, tout le monde était uni. [...] Puis, il y a eu une division politique. Lors de la campagne de 2011, tous les partis sont venus dire qu'ils allaient sauver Gabès de la pollution, et après ils ont disparu » (entretiens 2013).

Depuis la Révolution, la question des modes de valorisation des ressources locales s'est imposée au centre des débats politiques. Pourtant, elle n'a que peu donné lieu à une inflexion des politiques (Gana, 2013). Entre patrimonialisation et reproduction de modèles hérités, les oasis incarnent ces dynamiques contradictoires de reconfiguration des modèles de développement local, entre permanence et mutations.

\section{Conclusion}

En conclusion, la diversification des pratiques de l'agriculture oasienne et la différenciation sociale qui en résulte, loin d'être le signe d'une décomposition du territoire, sont plutôt la marque de dynamiques sociales vivaces, symboles d'un potentiel de résistance des systèmes oasiens à un déclin annoncé.

Le territoire local oasien se présente comme un atout pour les acteurs qui le contrôlent et tentent de le promouvoir, jouant ainsi, " avec plus ou moins de talent, le grand jeu de la mondialisation " (Théry, 2008). La multiplication des collectifs associatifs et des discours pour la sauvegarde des oasis, plutôt que de représenter une véritable transition des modèles, est le signe de l'irruption d'un nouvel enjeu politique oasien. L'intégration et la valorisation des espaces sahariens du sud de la Tunisie constituent des enjeux qui rappellent la dimension proprement politique de l'ouverture d'un débat sur les modèles de développement, impliquant exploitants, acteurs associatifs et institutions.

Dans un contexte de transition démocratique, l'analyse de ces dynamiques montre qu'il ne peut être question uniquement de sauvegarder un milieu, démarche finalement assez réductrice dans une réflexion sur la durabilité, dans la mesure où elle a tendance à valoriser les permanences au détriment des 
dynamiques de territoires. C'est plutôt la question de la place des territoires ruraux dans le développement national qui est en jeu. Les dynamiques contemporaines, en réactivant la plurifonctionnalité des territoires oasiens, leurs garantissent le rôle historique d'espace pivots, de mise en contact des aires urbaines, steppiques et agricoles. C'est l'affirmation d'un territoire d'action au cœur des nouveaux enjeux du développement au Sud.

\section{Références}

Abaab A. 2004. L'oasis de Gabès, une oasis en pleine mutation : Quel avenir pour l'élevage bovin laitier ? Projet d'intensification de l'élevage caprin dans le sud tunisien. Rapport de mission, CIHEAM.

Abdedayem S, Veyrac-Ben Ahmed S. 2014. Le paradoxe des oasis du sud tunisien : rénovation ou mort d'un concept ? In : Actes du colloque Oasis dans la mondialisation : ruptures et continuités. Paris, France, pp. 21-28.

Abdedayem S. 2015. Stratégie et plan d'action de développement durable des oasis. GEF (Global Environnement Facily), WB (World Bank), GDEO (Gestion durable des écosystèmes oasiens en Tunisie), $150 \mathrm{p}$.

Bachta MS, Legal P-Y, Rhouma A, Kuper M. 2006. De 1'eau aux dattes : aperçu de la filière datte tunisienne et perspectives d'interventions. In : Économies d'eau en systèmes irrigués au Maghreb. Deuxième atelier régional du projet Sirma. Marrakech, Maroc. Disponible sur http://hal.cirad.fr/cirad-00270996.

Battesti V. 2005. Jardins au désert, évolution des pratiques et savoirs oasiens, Jérid tunisien. IRD Éditions, coll. À travers champs, 440 p.

Belhedi A. 1999. Les disparités spatiales en Tunisie, état des lieux et enjeux. Méditerrannée 91(1): 63-72.

Bras JP. 1996. L'autre Tunisie de Bourguiba : les ombres du Sud. In : Camau M, Geisser V, eds. Bourguiba, la trace et l'héritage, Karthala. Paris, pp. 295-312.

Campagne P, Pecqueur B. 2014. Le développement territorial. Une réponse émergente à la mondialisation. Éditions Charles Léopold Mayer, $268 \mathrm{p}$.

Carpentier I, Gana A. 2014. Les oasis de Tozeur et Chenini Gabès : diversité et durabilité des formes de valorisation à l'ère de la mondialisation et des crises du développement. In : Actes du colloque Oasis dans la mondialisation : ruptures et continuités. Paris, France, pp. 105-112.

Carpentier I, Gana A. 2017. Changing agricultural practices in the oases of southern Tunisia: conflict and competition for resources in a post-revolutionary and globalisation context. In: Lavie E, Marshall A, eds. Oases and globalization, ruptures and continuities. Springer.

Côte M. 2002. Des oasis aux zones de mise en valeur, l'étonnant renouveau de l'agriculture saharienne. Méditerrannée 99(3-4): $5-14$.

D’Aquino P. 2002. Le territoire entre espace et pouvoir : pour une planification territoriale ascendante. L'Espace géographique 31: 3-22.

Di Méo. 2006. Les territoires de l'action. Bulletin de la Société géographique de Liège: 7-17.

FAO, 2008. Conservation et aménagement durable des SIPAM, (Systèmes ingénieux du patrimoine agricole mondial), asmgafsa. org

FEM (Fond mondial pour l'environnement), ministère de l'Environnement. 2015. Plan d'action de la stratégie de développement durable des oasis en Tunisie. Disponible sur http://www. environnement.gov.tn/fileadmin/medias/pdfs/projet_etude/pro jet_GDEO/3_2.pdf.

Gachet J-P. 1987. L'agriculture, discours et stratégies. In : Camau M, ed. Tunisie, une modernité au-dessus de tout soupçon? Aixen-Provence : IREMAM, CNRS, pp. 181-228.

Gana A. 2008. Restructurations agricoles en Tunisie : adaptations et différenciation. Autrepart 2(46): 81-96.

Gana A. 2013. Aux origines rurales et agricoles de la révolution tunisienne. Maghreb-Machrek 1(215): 57-80.

GIZ, ministère de l'Environnement tunisien. 2012. Les oasis de Tunisie à protéger contre la dégradation et les effets du changement climatique. 34 p. Disponible sur http://www.environnement.gov.tn/ PICC/wp-content/uploads/Les-oasis-face-au-changement-clima tique.pdf.

Ministère de l'Environnement et du Développement durable tunisien, Direction générale de l'environnement et de la qualité de la vie. 2015. Stratégie de développement durable des Oasis en Tunisie, 184 p.

Pecqueur B. 2006. Le tournant territorial de l'économie globale. Espaces et sociétés 2(124-125): 17-32.

Romdhane A, Abdeladhim M. 2008. Évolution des systèmes oasiens et comportements des exploitants agricoles « Cas des oasis littorales. Sud-est tunisien $»$.

Théry H. 2008. Mondialisation, déterritorialisation, reterritorialisation (Globalization and territory). Bulletin de l'Association des géographes français, $85^{\mathrm{e}}$ année, 2008-3 (septembre). Mondialisation et géographie/L'ouest américain, pp. 324-331.

Torre A. 2015. Théorie du développement territorial. Géographie, économie, société 3(17): 273-288.

Citation de l'article : Carpentier I. 2017. Diversité des dynamiques locales dans les oasis du Sud de la Tunisie. Cah. Agric. $26: 35001$. 